\title{
Social media posts and online search behaviour as early-warning system for MRSA outbreaks
}

Tom H. van de Belt ${ }^{1 *}$ (D, Pieter T. van Stockum², Lucien J. L. P. G. Engelen ${ }^{1}$, Jules Lancee ${ }^{1}$, Remco Schrijver ${ }^{2}$, Jesús Rodríguez-Baño ${ }^{3}$, Evelina Tacconellii, ${ }^{4,5}$, Katja Saris ${ }^{6,8}$, Marleen M. H. J. van Gelder ${ }^{1,7}$ and Andreas Voss ${ }^{1,6,8}$

\begin{abstract}
Background: Despite many preventive measures, outbreaks with multi-drug resistant micro-organisms (MDROs) still occur. Moreover, current alert systems from healthcare organizations have shortcomings due to delayed or incomplete notifications, which may amplify the spread of MDROs by introducing infected patients into a new healthcare setting and institutions. Additional sources of information about upcoming and current outbreaks, may help to prevent further spread of MDROs.

The study objective was to evaluate whether methicillin-resistant Staphylococcus aureus (MRSA) outbreaks could be detected via social media posts or online search behaviour; if so, this might allow earlier detection than the official notifications by healthcare organizations.
\end{abstract}

Methods: We conducted an exploratory study in which we compared information about MRSA outbreaks in the Netherlands derived from two online sources, Coosto for Social Media, and Google Trends for search behaviour, to the mandatory Dutch outbreak notification system (SO-ZI/AMR). The latter provides information on MDRO outbreaks including the date of the outbreak, micro-organism involved, the region/location, and the type of health care organization.

Results: During the research period of 15 months (455 days), 49 notifications of outbreaks were recorded in SO-ZI/ AMR. For Coosto, the number of unique potential outbreaks was 37 and for Google Trends 24. The use of social media and online search behaviour missed many of the hospital outbreaks that were reported to SO-ZI/AMR, but detected additional outbreaks in long-term care facilities.

Conclusions: Despite several limitations, using information from social media and online search behaviour allows rapid identification of potential MRSA outbreaks, especially in healthcare settings with a low notification compliance. When combined in an automated system with real-time updates, this approach might increase early discovery and subsequent implementation of preventive measures.

Keywords: Methicillin-resistant Staphylococcus aureus, MRSA, Social media monitoring, Outbreaks, Google trends, Nowcasting

\footnotetext{
* Correspondence: tom.vandebelt@radboudumc.nl

${ }^{1}$ Radboud REshape Innovation Center, Radboudumc University Medical

Center, Geert Grooteplein Zuid 10, 6525, GA, Nijmegen, Netherlands

Full list of author information is available at the end of the article
}

(c) The Author(s). 2018 Open Access This article is distributed under the terms of the Creative Commons Attribution 4.0 International License (http://creativecommons.org/licenses/by/4.0/), which permits unrestricted use, distribution, and reproduction in any medium, provided you give appropriate credit to the original author(s) and the source, provide a link to the Creative Commons license, and indicate if changes were made. The Creative Commons Public Domain Dedication waiver (http://creativecommons.org/publicdomain/zero/1.0/) applies to the data made available in this article, unless otherwise stated. 


\section{Background}

The Dutch healthcare system applies strict infection control guidelines regarding multi-drug resistant micro-organisms (MDROs), including the "Search \& Destroy" guideline for methicillin-resistant Staphylococcus aureus (MRSA), which was extended to other MDROs in 2011 [1, 2]. Despite the implementation of these guidelines, outbreaks with MDROs still occur. Reasons may be a temporary lack of compliance with existing guidelines, human error, or spread from infected patients not falling into a high-risk category that would warrant screening and isolation on admission. One of the defined high-risk-categories of the Dutch MRSA/MDRO guideline, are patients originating from a healthcare setting with an ongoing MRSA/MDRO outbreak. In the past, hospitals were supposed to inform each other about outbreaks and possible colonized or infected patients they exchange, but the report itself as well as the way of communication were non-standardized and voluntarily. As of 2012, all hospitals report their MDRO outbreaks to a central point (SO-ZI/ AMR), which was initiated and established by the Dutch Society of Clinical Microbiology (NVMM) after the first carbapenem-resistant Enterobacteriaceae (CRE)-outbreak in the Netherlands [3]. SO-ZI/AMR contains a database with information about the outbreak such as date and duration of the outbreak, organization name affected location(s) and the micro-organism in question. Outbreaks that need to be reported to SO-ZI/AMR are defined as: Outbreaks which influence, or have the potential to negatively influence, access to care, such as in case of (possible) closure of a department or part of it, and/or outbreaks with continuous transmission despite (infection) control measures [4].

When reporting outbreaks became part of the professional guidelines, it became essentially mandatory. However, reporting outbreaks is currently only mandatory for hospitals, and not for nursing homes or other health care institutions. Once reported, an outbreak is, with a short delay, immediately visible for all users. The task of SO-ZI/AMR is not only to collect reports and report outbreaks to professionals, but also to monitor the development of the outbreak, and, if needed, to support the control efforts. Still, the alert messages from some hospitals seem to come late or not at all, with the risk of introducing patients infected with an MDRO into a new healthcare setting without warning and increasing the probability of spreading the outbreak. Therefore, there is a need for additional sources of information about current and potentially upcoming outbreaks, to increase outbreak preparedness.

Since an increasing number of people use social media, such as Facebook and Twitter, to share information and the Internet as source for news, social media posts and online search behaviour (e.g., via search engines) could be a valuable source of information about potential MDRO outbreaks. Interestingly, online search behaviour has already successfully been used to detect influenza outbreaks based on search entries [5], and disease outbreaks in general [6]. Moreover, social media have been used to monitor the quality of healthcare institutions on, for example, hygiene and expertise [7]. The aim of the current study was to evaluate whether Dutch MRSA outbreaks could be detected via social media posts or online search behaviour; and if so, whether these data sources might allow earlier detection than the official notification to SO-ZI/AMR by the hospital. In addition, as reporting outbreaks in nursing homes is still voluntary, we also evaluated whether screening of social media posts and/or online search behaviour would help to identify outbreaks in these healthcare institutions. In this study, we focused on MRSA.

\section{Methods \\ Design and Setting}

We conducted an exploratory study in which we compared information about MRSA outbreaks derived from social media and online search behaviour to the official Dutch reference standard. MRSA specific searches were performed for the time period between January 1st, 2015 and March 31st, 2017. As reference standard for MRSA outbreaks, SO-ZI/AMR was used [8]. It provides information on official outbreaks including the date of the outbreak and the region and type of health care facility (e.g. hospital or nursing home). The geographical scope of the study was The Netherlands; therefore, we only searched using the Dutch language.

\section{Social media sources and online search behaviour}

To capture social media posts about MRSA (all publicly shared social media posts by individuals or organizations), we used Coosto, a social media monitoring tool [9]. This tool has proven to be a valuable source of social media information and is currently in use by the Dutch government to monitor the quality of healthcare organizations [7]. It provides the exact time and, if available, the location of the message in various social media sources, including Facebook and Twitter. Presently, its database includes posts in the Dutch language.

In addition, Google Trends was used to assess online search behaviour [10]. This tool provides insight into the search behaviour based on specific searches performed in Google Search. It provides the relative frequency of searches for different countries and regions. Google Trends has been used for early detection of influenza outbreaks [6]. Although multiple search engines are being used in The Netherlands, we limited our searches to Google Trends since Google covers over 80\% of Internet 
searches, capturing the overall majority of Internet searches [11].

\section{Data extraction}

We searched Coosto for publicly available social media posts about MRSA with the following search query: ("mrsa" OR "methicillin-resistant Staphylococcus aureus" OR "methicillin resistant Staphylococcus aureus" OR "meticilline-resistente Staphylococcus aureus" OR "meticilline resistente Staphylococcus aureus"). Several preliminary searches revealed that both Dutch and English names had to be included, since English terminology was sometimes used in Dutch social media posts and the combination maximized the number of hits. Furthermore, the word 'outbreak' was not included in the search query, since the preliminary searches showed that searching for 'outbreak' resulted in a large number of irrelevant hits, and that combining 'outbreak' with MRSA via Boolean search ("AND”) negatively affected the sensitivity of the search. Based on the results of the preliminary searches, we performed manual inspections of results with 25, 15 and 10 hits per day. In general, the lowest number of hits per day would result the highest number of potential outbreaks. However, from the above comparison, we concluded found that 10 posts per day was the minimum number of hits, or 'critical mass' [7] to identify a potential MRSA outbreak, Consequently, we set ( $\geq 10$ hits) as criterion for a potential outbreak. For all posts on a specific day, that met this criterion, we identified whether a potential outbreak was discussed, meaning that MRSA was mentioned in relation to a Dutch healthcare institution and indicating a present or potential outbreak. The latter could consist of (but not limited to) patients, relatives or employees found or suspected with MRSA, hospital wards closed due to MRSA, any other information about a present or expected MRSA outbreak shared by the institution, its employees, government, or other any other individual (e.g., patients or relatives). Days meeting all criteria were marked as 'representing a potential MRSA outbreak' and all other days as 'not representing a potential MRSA outbreak'. Dates, number of hits, institution and geographical area and outbreak (YES/NO) were subsequently stored in a research database.

Regarding the searches in Google Trends, we used similar terms, but with individual searches for each term. Google Trends presents search interest of topics on a scale from 0 to 100 per day instead of the absolute number of searches, thus every search will have at least one day with the maximum score of 100 , even when absolute search numbers are low during a particular time period (e.g., a time period without any outbreaks). This system characteristic required a different way of defining days with potential outbreaks. Assuming that a Dutch MRSA outbreak occurred at least once every 3 months, we used the mean search interest of 3 months in our analyses. We extracted search interest per day, as well as geographical information (province) for days with potential outbreaks.

\section{Statistical analysis}

All statistical analyses were done in SPSS version 22. The database with search results from Coosto and Google Trends was compared to data from the official SO-ZI/AMR database. In case of consecutive days with the same potential outbreak, we defined this as a single outbreak, both for Coosto and Google Trends. To assess the validity of Coosto to detect potential MRSA outbreaks, we calculated the overall sensitivity, specificity, positive predictive value (PPV), and negative predictive value (NPV) with 95\% confidence intervals (CIs). Furthermore, we stratified the analyses by type of healthcare institution affected.

For the Google Trends data, a search score was calculated for each day:

Google Trends Score $=($ relative search volume - mean relative search volume) / standard deviation.

with the mean relative search volume and standard deviation based on the preceding 3 months. Using SO-ZI/ AMR as the reference standard, we calculated the area under the curve (AUC) for 7 days before until 7 days after an outbreak. Furthermore, as the optimal cut-off value to detect an outbreak based on online search behaviour is unknown, we determined the Google Trends Score that maximized the sum of sensitivity and specificity. For the present study, we used a cut-off value of $2 *$ SD to detect a potential outbreak with Google Trends. Finally, we calculated the Pearson correlation coefficient

Table 1 Characteristics of MRSA outbreaks detected in SO-ZI/ AMR and Coosto

\begin{tabular}{|c|c|c|c|}
\hline & SO-ZI/AMR & Coosto & Google Trends \\
\hline Number of days with $\geq 10$ posts & & 297 & \\
\hline Excluded & & 260 & \\
\hline Multiple days & & 22 & \\
\hline New research & & 33 & \\
\hline Unrelated messages & & 59 & \\
\hline General info concerning MRSA & & 115 & \\
\hline Articles about hygiene and meat & & 31 & \\
\hline Total number of outbreaks & 49 & 37 & 24 \\
\hline University hospital & 8 & 1 & \\
\hline General hospital & 22 & 17 & \\
\hline Nursing home & 7 & 16 & \\
\hline Other & 3 & 3 & \\
\hline Unknown & 9 & 0 & \\
\hline
\end{tabular}




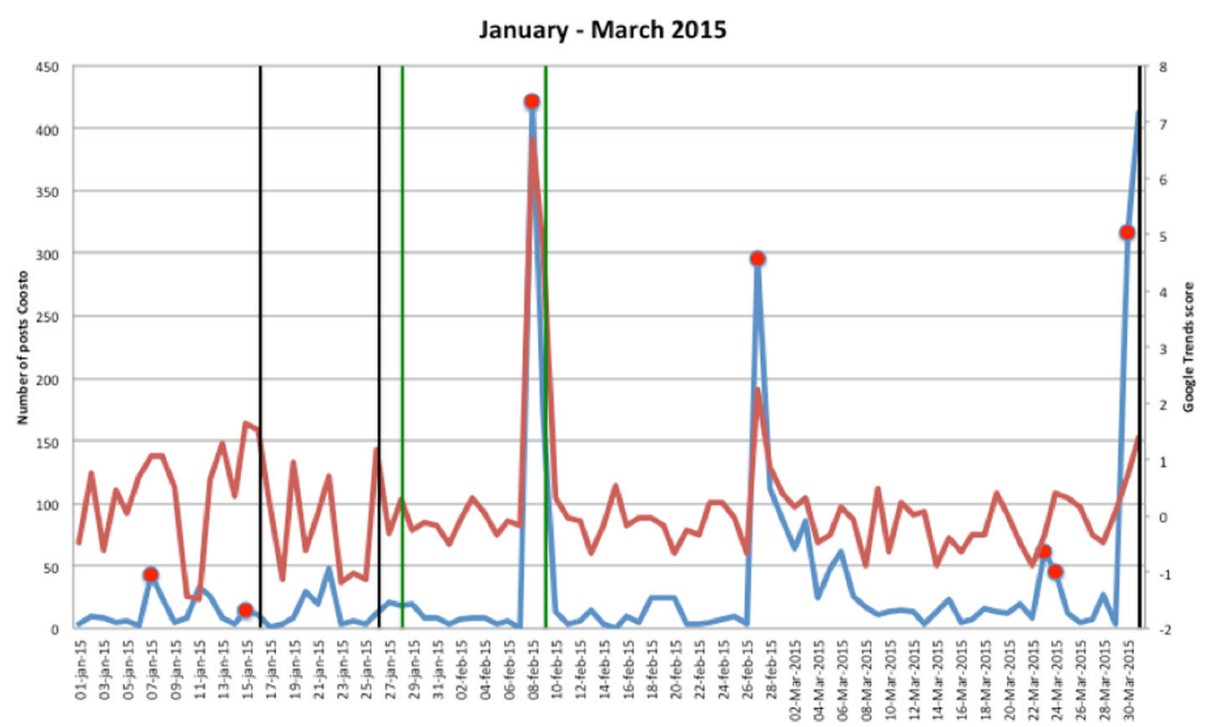

Fig. 1 MRSA outbreaks in The Netherlands, per quarter of a year. The blue line is the number related to MRSA detected by Coosto, with a red dot indicating days with $\geq 10$ posts related to an outbreak, the red line the Google Trends score. The vertical bars represent outbreaks reported to SO-ZI/AMR: blue for teaching hospitals, black for regular hospitals, green for nursing homes, yellow for other locations, and red for unknown locations

to assess the association between the number of posts related to MRSA detected with Coosto and the Google Trends Score.

\section{Results}

During the research period of 15 months (455 days), 49 outbreaks were reported to SO-ZI/AMR (Table 1). Using Coosto, 37 potential outbreaks were detected based on social media posts, of which 1 referred to an academic hospital, 17 to a general hospital, 16 to a nursing home, and 3 to other types of institutions. Google Trends resulted in 24 potential outbreaks.

Figures 1, 2, 3, 4, 5, 6, 7, 8, and 9 show the information on MRSA outbreaks originating from the three data sources in each quarter of a year. In only 4 outbreaks did all three sources show a (potential) outbreak, with in

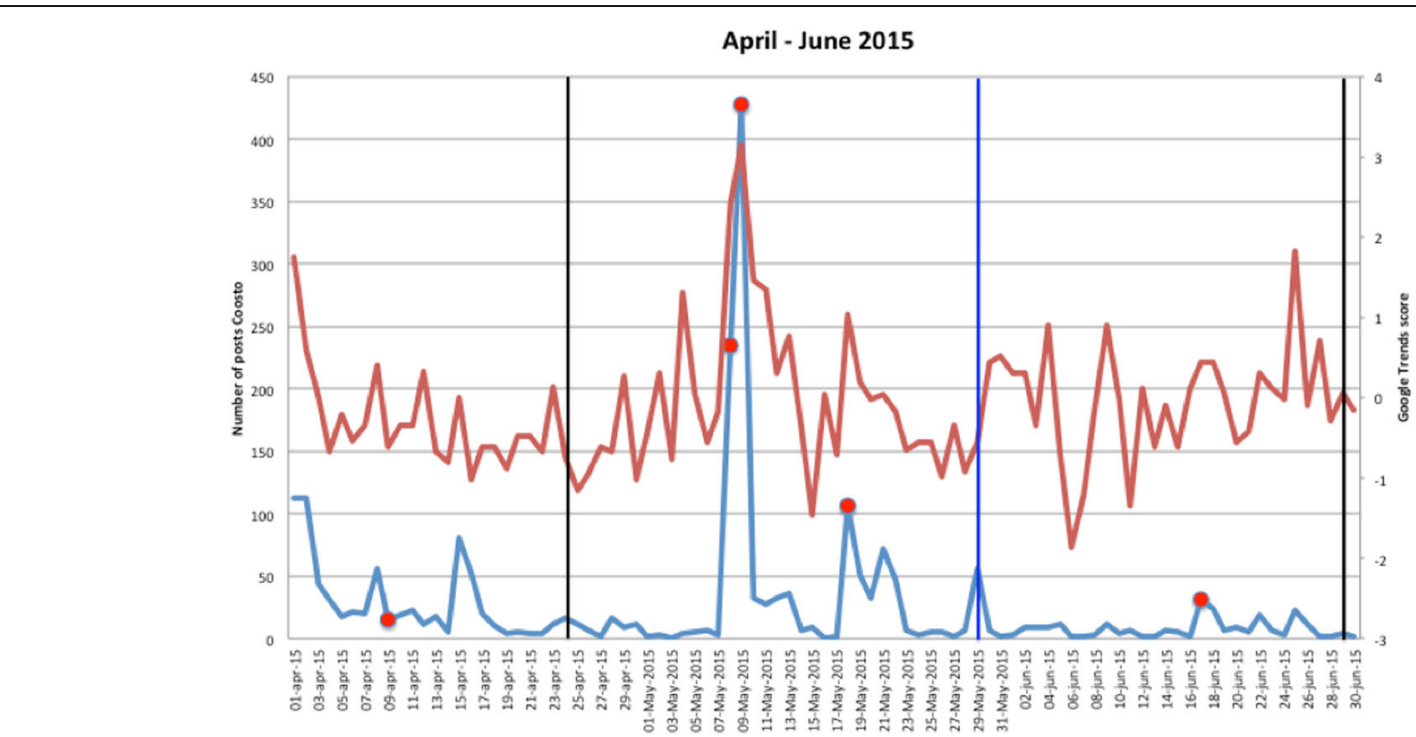

Fig. 2 MRSA outbreaks in The Netherlands, per quarter of a year. The blue line is the number related to MRSA detected by Coosto, with a red dot indicating days with $\geq 10$ posts related to an outbreak, the red line the Google Trends score. The vertical bars represent outbreaks reported to SO-ZI/AMR: blue for teaching hospitals, black for regular hospitals, green for nursing homes, yellow for other locations, and red for unknown locations 


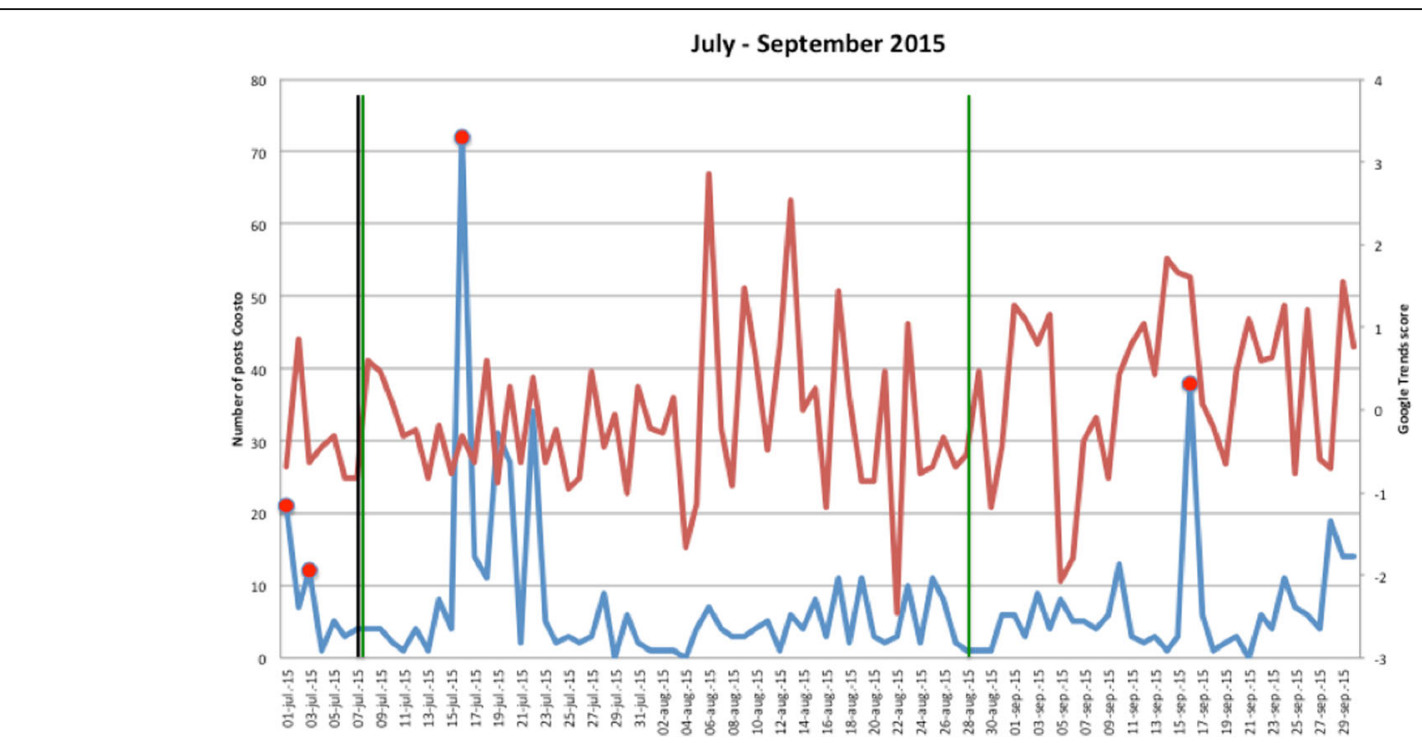

Fig. 3 MRSA outbreaks in The Netherlands, per quarter of a year. The blue line is the number related to MRSA detected by Coosto, with a red dot indicating days with $\geq 10$ posts related to an outbreak, the red line the Google Trends score. The vertical bars represent outbreaks reported to SO-ZI/AMR: blue for teaching hospitals, black for regular hospitals, green for nursing homes, yellow for other locations, and red for unknown locations

general the online sources detecting the outbreak 12 days before the official notification date in SO-ZI/ AMR. In 48 cases, Coosto and/or Google Trends indicated a (potential) outbreak without notification in SO-ZI/AMR, whereas in 41 cases, MRSA outbreaks were notified to SO-ZI/AMR that went unnoticed by the online data sources. In 4 cases, a (potential) outbreak was detected by both SO-ZI-AMR and Coosto, and not by Google Trends. Correlation between the number of posts related to MRSA detected with Coosto and the Google Trends Score was 0.42, $p<0.001$ ).

Validity comparisons for Coosto-detected MRSA outbreaks showed an overall sensitivity of 0.20 (95\% CI 0.10 0.34 ) and an overall specificity of 0.96 (95\% CI $0.95-0.98)$, whereas the PPV and NPV were 0.27 (95\% CI 0.16-0.42) and 0.95 (95\% CI 0.92-0.96), respectively (Table 2). After

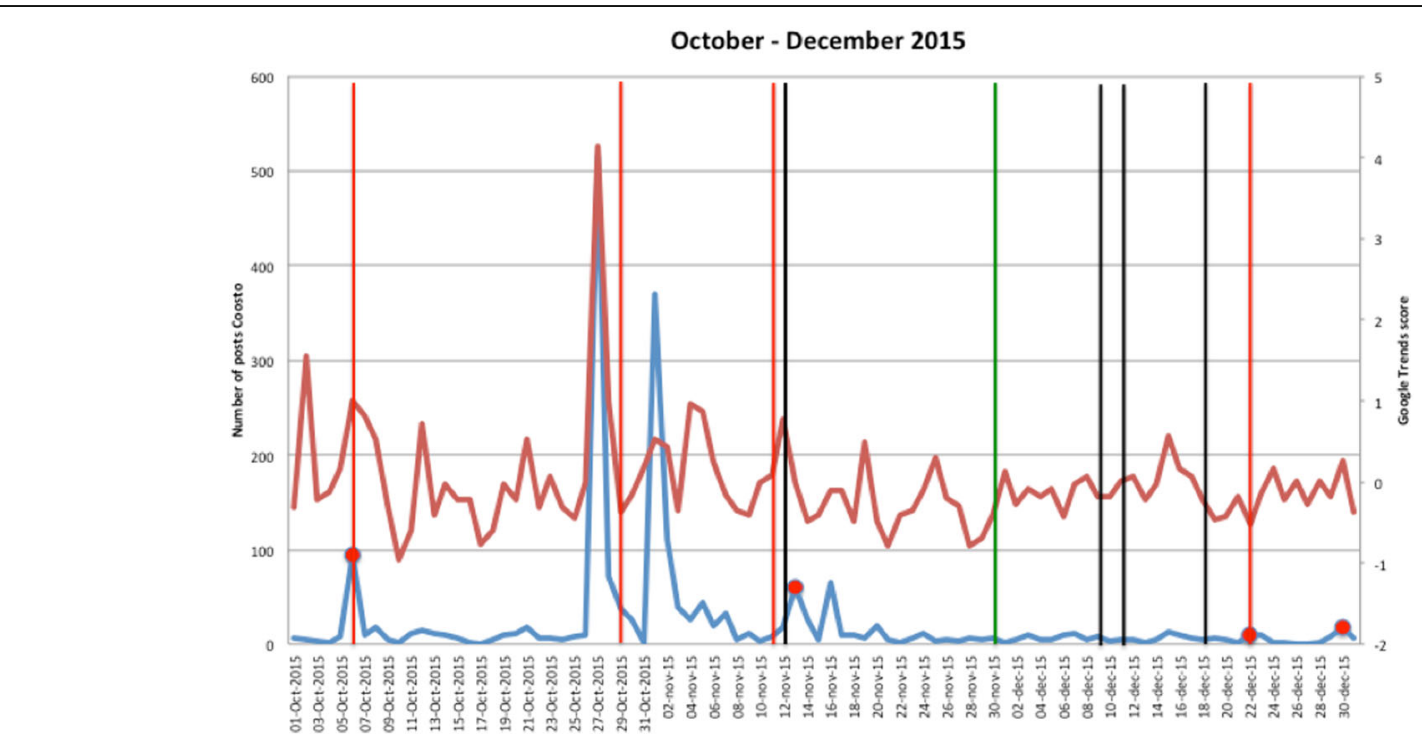

Fig. 4 MRSA outbreaks in The Netherlands, per quarter of a year. The blue line is the number related to MRSA detected by Coosto, with a red dot indicating days with $\geq 10$ posts related to an outbreak, the red line the Google Trends score. The vertical bars represent outbreaks reported to SO-ZI/AMR: blue for teaching hospitals, black for regular hospitals, green for nursing homes, yellow for other locations, and red for unknown locations 


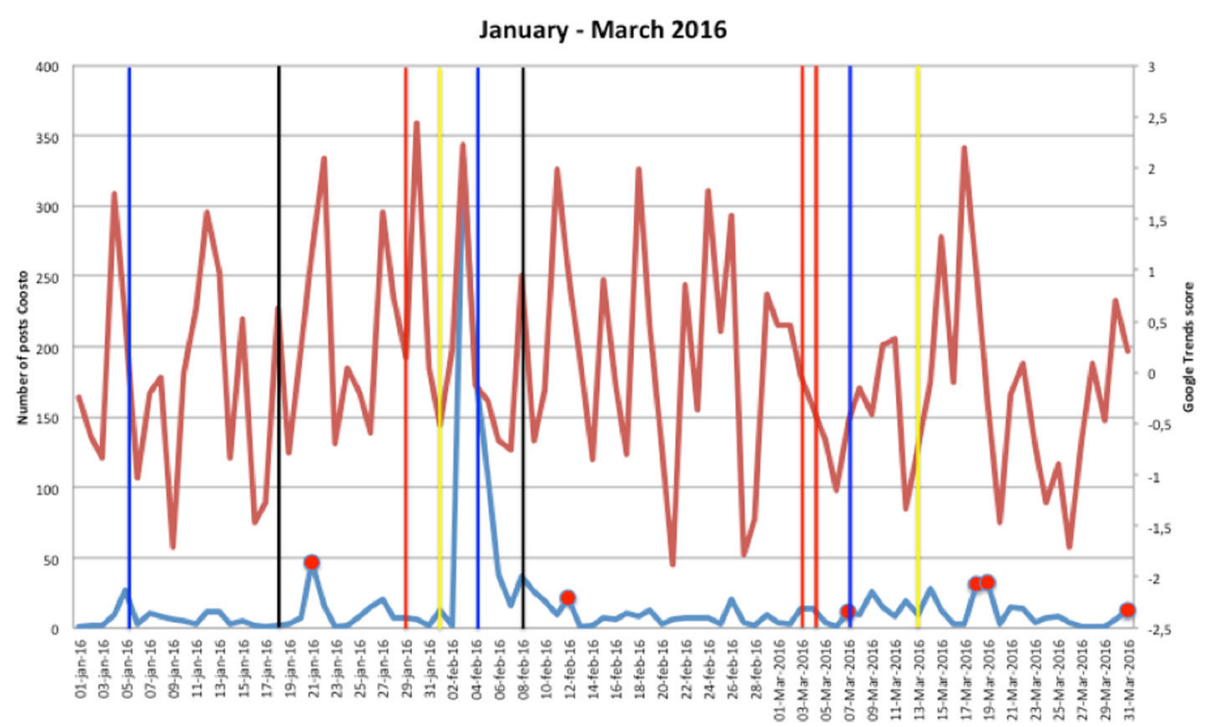

Fig. 5 MRSA outbreaks in The Netherlands, per quarter of a year. The blue line is the number related to MRSA detected by Coosto, with a red dot indicating days with $\geq 10$ posts related to an outbreak, the red line the Google Trends score. The vertical bars represent outbreaks reported to SO-ZI/AMR: blue for teaching hospitals, black for regular hospitals, green for nursing homes, yellow for other locations, and red for unknown locations

stratification for type of healthcare institution, sensitivity ranged between 0 for other and unknown locations to 0.23 (95\% CI 0.08-0.45) for general hospitals. Specificity was $\geq 0.98$ for all types of institutions.

The validity comparisons for Google Trends to detect MRSA outbreaks compared with SO-ZI/AMR as the reference standard are shown in Table 3. On the exact date the outbreak was notified to SO-ZI/AMR, the AUC was 0.59 (95\% CI 0.51-0.67) for any MRSA outbreak and
0.63 (95\% CI 0.54-0.73) for MRSA outbreaks in hospitals. With the optimal cut-off for the Google Trends Score, sensitivity was higher for any outbreak compared with hospital outbreaks only ( 0.90 vs. 0.43$)$, whereas specificity was higher for hospital outbreaks ( 0.28 vs. 0.79$)$. The AUC based on the Google Trends Score 1 day before the official notification was similar to the AUC on the day of notification. On the other days relative to the notification of the outbreaks, the AUC was decreased.

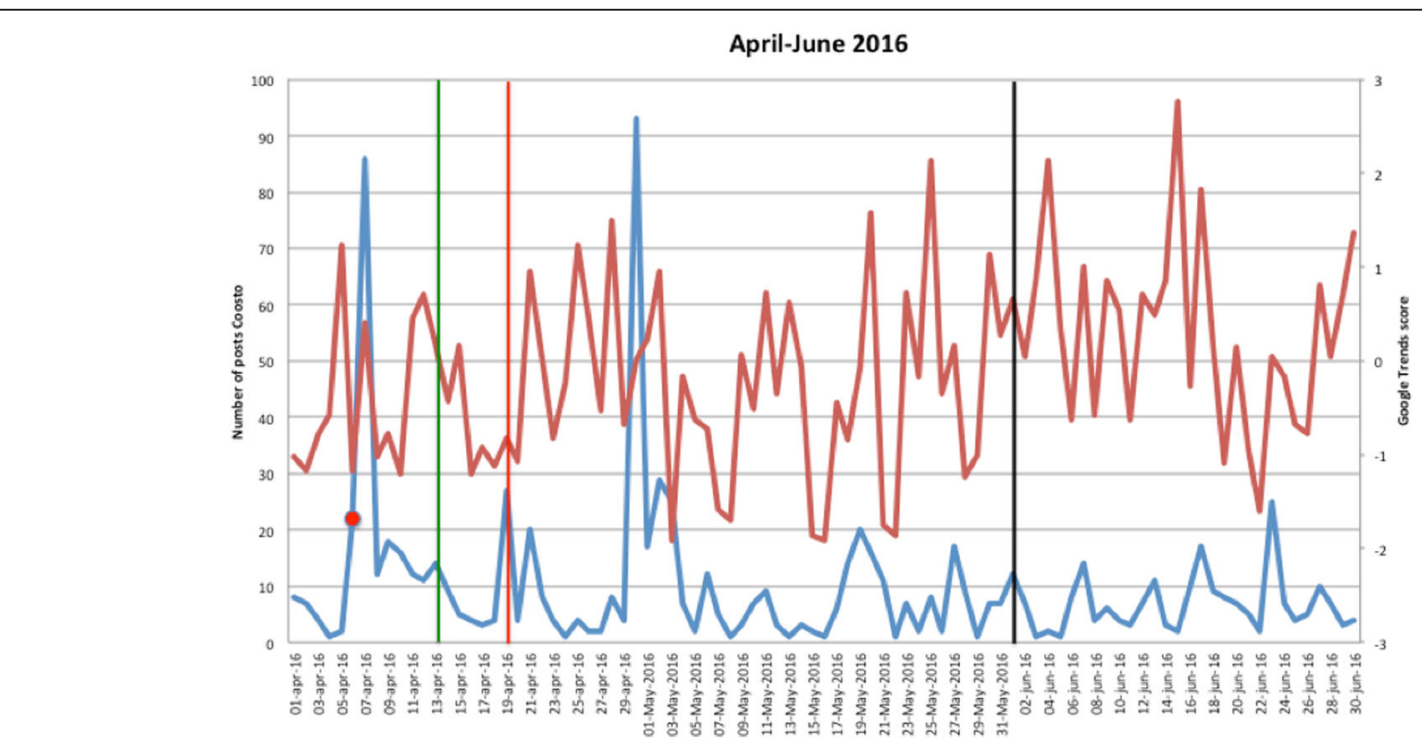

Fig. 6 MRSA outbreaks in The Netherlands, per quarter of a year. The blue line is the number related to MRSA detected by Coosto, with a red dot indicating days with $\geq 10$ posts related to an outbreak, the red line the Google Trends score. The vertical bars represent outbreaks reported to SO-ZI/ AMR: blue for teaching hospitals, black for regular hospitals, green for nursing homes, yellow for other locations, and red for unknown locations 


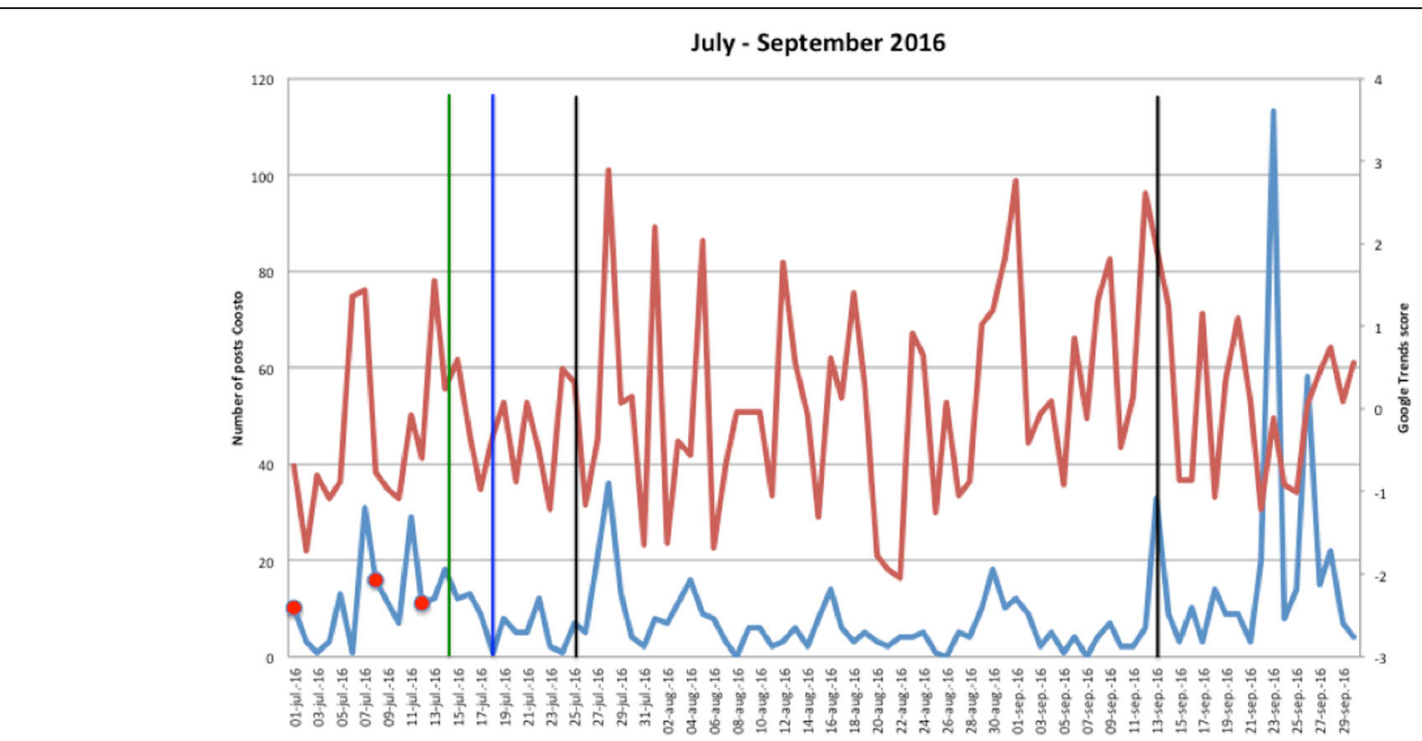

Fig. 7 MRSA outbreaks in The Netherlands, per quarter of a year. The blue line is the number related to MRSA detected by Coosto, with a red dot indicating days with $\geq 10$ posts related to an outbreak, the red line the Google Trends score. The vertical bars represent outbreaks reported to SO-ZI/ AMR: blue for teaching hospitals, black for regular hospitals, green for nursing homes, yellow for other locations, and red for unknown locations

\section{Discussion}

\section{Principal findings}

In this study, we compared information about potential MRSA outbreaks retrieved from social media posts and online search behaviour in The Netherlands to the national notification reference standard. We found that simple online (social media) searches do provide additional information about potential MRSA outbreaks in The Netherlands compared to the reference standard.
These promising findings suggest that supervisory bodies such as $\mathrm{SO}-\mathrm{ZI} / \mathrm{AMR}$ may enrich their palette of data sources with more dynamic information from social media and other online sources such as search engine data. However, the validity of the online sources Coosto and Google trends needs further investigation. Some things need to be discussed.

The sensitivity of the social media monitoring tool Coosto to detect MRSA outbreaks was low and therefore a

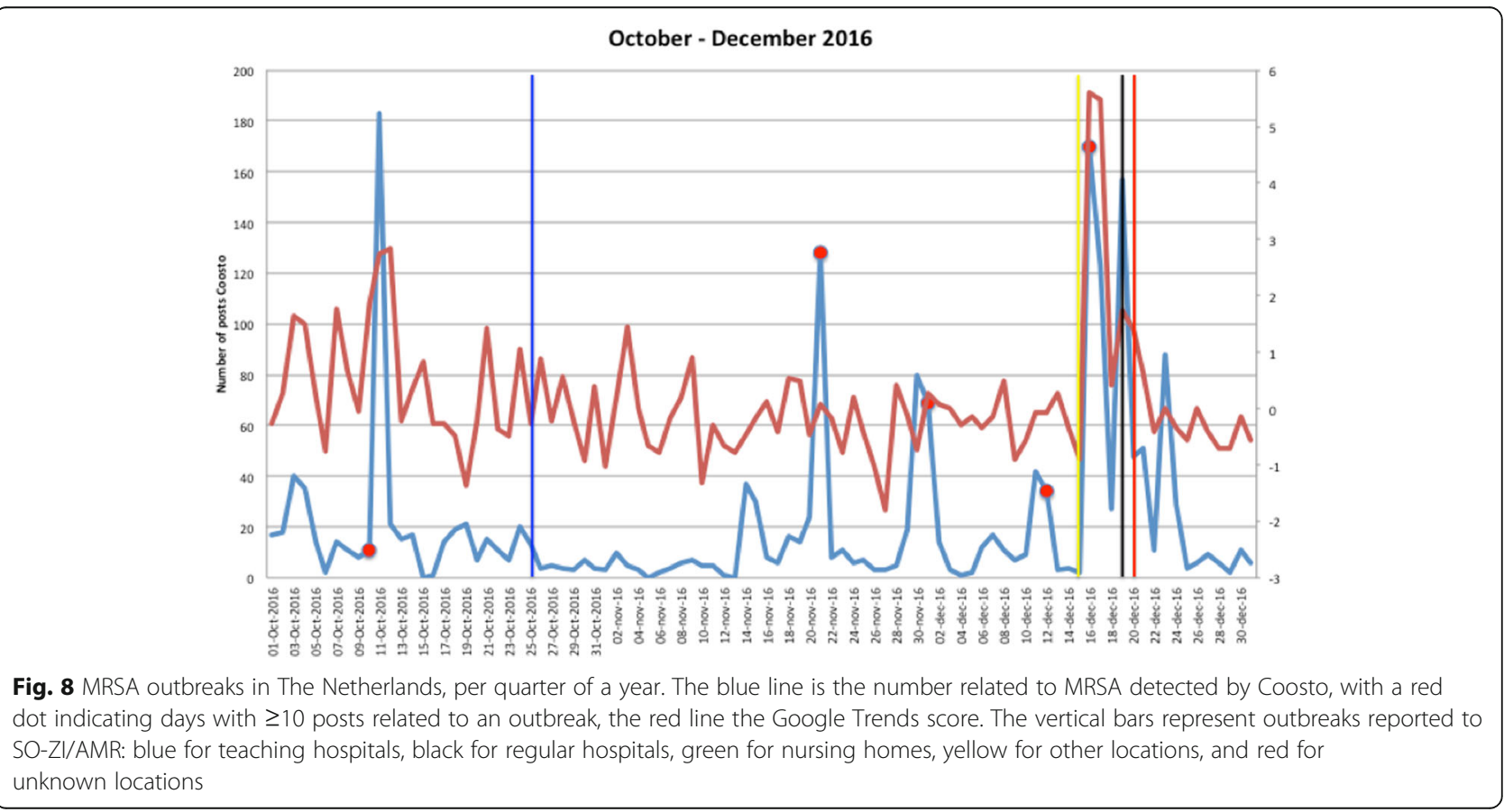




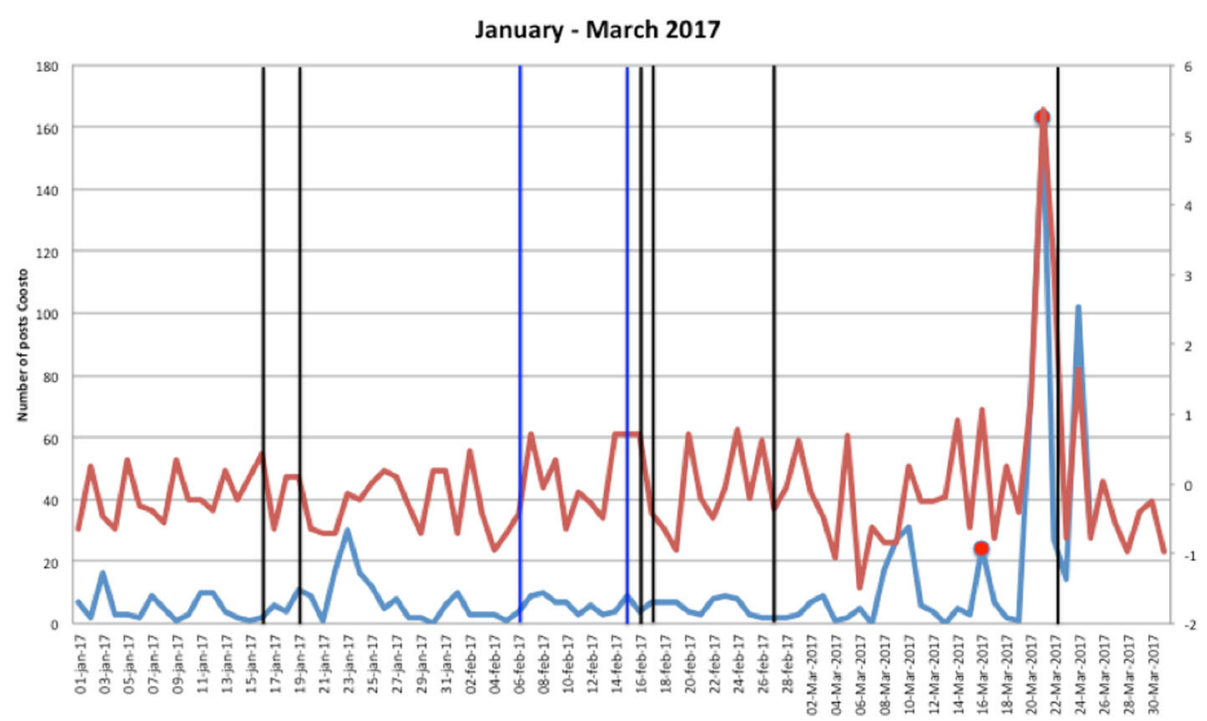

Fig. 9 MRSA outbreaks in The Netherlands, per quarter of a year. The blue line is the number related to MRSA detected by Coosto, with a red dot indicating days with $\geq 10$ posts related to an outbreak, the red line the Google Trends score. The vertical bars represent outbreaks reported to SO-ZI/AMR: blue for teaching hospitals, black for regular hospitals, green for nursing homes, yellow for other locations, and red for unknown locations

substantial number of true outbreaks will be missed when relying on this data source. However, its specificity was high, indicating a relatively small number of false positive outbreaks detected by Coosto. Interestingly, the opposite was observed for Google Trends, with a higher sensitivity and lower specificity, indicating that Google Trends detects more potential MRSA outbreaks but that many of these represent false positive signals. This difference between the two online data sources may be explained by their nature: social media posts provide more detailed information on MRSA outbreaks than online searches, but the patients and healthcare workers involved in MRSA outbreaks may be more reluctant to post a message about the outbreak on social media than to search for information online. In addition, it is impossible to distinguish between online searches for actual outbreaks and searches for random issues related to MRSA using Google Trends.

To the best of our knowledge, this was the first study using online search engines for social media posts and internet search behaviour on MRSA outbreak detection. A study by Lui et al. used search terms from the social media platform Baidu to identify Noro virus epidemics [12]. They concluded that several limitations exist to using Internet to monitor epidemics but that it still

Table 2 Validity comparisons for Coosto-detected MRSA outbreaks with SO-ZI/AMR as the reference standard

\begin{tabular}{|c|c|c|c|c|c|c|c|c|c|c|c|c|}
\hline \multirow[t]{2}{*}{ MRSA outbreaks } & \multicolumn{4}{|c|}{ Number of days } & \multicolumn{8}{|c|}{ Validity } \\
\hline & $\overline{\mathrm{TP}}$ & FP & FN & $\overline{\mathrm{TN}}$ & $\mathrm{Se}$ & $(95 \% \mathrm{Cl})$ & $S p$ & $(95 \% \mathrm{Cl})$ & PPV & $(95 \% \mathrm{Cl})$ & NPV & $(95 \% \mathrm{Cl})$ \\
\hline All & 10 & 27 & 39 & 722 & 20 & $(10-34)$ & 96 & (95-98) & 27 & $(16-42)$ & 95 & (92-96) \\
\hline Any hospital & 5 & 13 & 25 & 755 & 17 & $(6-35)$ & 98 & (97-99) & 28 & $(13-50)$ & 97 & $(96-97)$ \\
\hline University hospital & 0 & 1 & 8 & 791 & 0 & & 100 & $(99-100)$ & 0 & & 99 & (99-99) \\
\hline General hospital & 5 & 12 & 17 & 764 & 23 & $(8-45)$ & 98 & (97-99) & 29 & $(14-52)$ & 98 & (97-98) \\
\hline Any hospital and unknown location & 6 & 12 & 33 & 747 & 15 & $(6-31)$ & 98 & $(97-99)$ & 33 & $(17-56)$ & 96 & $(95-96)$ \\
\hline University hospital and unknown location & 0 & 1 & 17 & 783 & 0 & & 100 & $(99-100)$ & 0 & & 98 & $(98-98)$ \\
\hline General hospital and unknown location & 6 & 11 & 25 & 756 & 19 & $(7-37)$ & 99 & $(97-99)$ & 35 & $(18-58)$ & 97 & $(96-97)$ \\
\hline Nursing home & 1 & 15 & 6 & 778 & 13 & $(0-53)$ & 98 & $(97-99)$ & 6 & $(1-31)$ & 99 & $(99-99)$ \\
\hline Nursing home and unknown location & 2 & 14 & 14 & 770 & 13 & $(2-38)$ & 98 & $(97-99)$ & 13 & $(3-37)$ & 98 & $(98-99)$ \\
\hline Other location & 0 & 3 & 3 & 795 & 0 & & 100 & $(99-100)$ & 0 & & 100 & $(100-100)$ \\
\hline Other location and unknown location & 0 & 3 & 12 & 786 & 0 & & 100 & $(99-100)$ & 0 & & 99 & $(98-99)$ \\
\hline
\end{tabular}

$\mathrm{Cl}$ confidence interval, $F N$ false negative, $F P$ false positive, NPV negative predictive value, $P P V$ positive predictive value, Se sensitivity, $S p$ specificity, $T N$ true negative, $T P$ true positive 
Table 3 Validity comparisons for Google Trends to detect MRSA outbreaks with SO-ZI/AMR as the reference standard. Day 0 indicates the day the outbreak was reported to SO-ZI/AMR

\begin{tabular}{|c|c|c|c|c|c|c|c|c|c|c|}
\hline \multirow[t]{2}{*}{ Day } & \multicolumn{5}{|c|}{ Any MRSA outbreak } & \multicolumn{5}{|c|}{ MRSA outbreak in hospital } \\
\hline & $\overline{\text { AUC }}$ & $(95 \% \mathrm{Cl})$ & Optimal cutoff ${ }^{a}$ & $\mathrm{Se}$ & $\overline{S p}$ & $\overline{\mathrm{AUC}}$ & $(95 \% \mathrm{Cl})$ & Optimal cutoff ${ }^{a}$ & $\mathrm{Se}$ & $\overline{S p}$ \\
\hline-7 & 0.61 & $(0.54-0.69)$ & -0.2018 & 75 & 47 & 0.59 & $(0.51-0.67)$ & -0.6507 & 100 & 23 \\
\hline-6 & 0.51 & $(0.44-0.59)$ & -0.1208 & 63 & 51 & 0.51 & $(0.42-0.59)$ & -0.6965 & 93 & 21 \\
\hline-5 & 0.46 & $(0.38-0.55)$ & -0.4318 & 71 & 33 & 0.48 & $(0.39-0.58)$ & -0.8801 & 97 & 13 \\
\hline-4 & 0.50 & $(0.42-0.58)$ & -0.5524 & 81 & 28 & 0.53 & $(0.44-0.62)$ & -0.5524 & 87 & 28 \\
\hline-3 & 0.46 & $(0.38-0.54)$ & -0.5377 & 81 & 29 & 0.48 & $(0.39-0.58)$ & -0.5377 & 87 & 29 \\
\hline-2 & 0.52 & $(0.44-0.61)$ & 0.1445 & 42 & 65 & 0.47 & $(0.37-0.57)$ & -0.6828 & 83 & 21 \\
\hline-1 & 0.59 & $(0.50-0.67)$ & 0.0425 & 60 & 61 & 0.59 & $(0.47-0.71)$ & 0.0672 & 67 & 62 \\
\hline 0 & 0.59 & $(0.51-0.67)$ & -0.5524 & 90 & 28 & 0.63 & $(0.54-0.73)$ & 0.3316 & 43 & 79 \\
\hline+1 & 0.54 & $(0.46-0.63)$ & -0.1769 & 63 & 48 & 0.49 & $(0.39-0.59)$ & -1.1493 & 100 & 7 \\
\hline+2 & 0.49 & $(0.41-0.57)$ & -1.1833 & 100 & 6 & 0.46 & $(0.36-0.55)$ & -0.9484 & 97 & 11 \\
\hline+3 & 0.50 & $(0.43-0.58)$ & -0.5149 & 79 & 29 & 0.56 & $(0.46-0.66)$ & -0.1118 & 63 & 51 \\
\hline+4 & 0.49 & $(0.41-0.57)$ & -0.6828 & 90 & 21 & 0.49 & $(0.40-0.59)$ & -0.6828 & 93 & 21 \\
\hline+5 & 0.44 & $(0.37-0.51)$ & 0.1674 & 15 & 65 & 0.39 & $(0.31-0.47)$ & -1.3370 & 100 & 4 \\
\hline+6 & 0.49 & $(0.42-0.57)$ & -1.0128 & 98 & 9 & 0.47 & $(0.38-0.57)$ & -1.0128 & 97 & 9 \\
\hline+7 & 0.53 & $(0.45-0.60)$ & -0.4993 & 85 & 30 & 0.50 & $(0.40-0.61)$ & -0.5745 & 83 & 27 \\
\hline
\end{tabular}

AUC area under the curve, $\mathrm{Cl}$ confidence interval, Se sensitivity, $S p$ specificity

${ }^{a}$ Maximizes sensitivity+specificity. Value represents $\mathrm{k}$

might have value as additional tool, particularly when other monitoring systems are lacking. Also, the importance of social media as an early warning system in addition to traditional slow reporting mechanisms has been emphasized [13]. In general, this might be the case for all outbreaks as notification is only done after firm confirmation of the outbreak. Consequently, the notification date in SO-ZI/AMR is "delayed" by several days.

Additional potential outbreaks were found via social media, which were not in the SO-ZI/AMR database. Most of these outbreaks occurred in nursing homes for which notification is not mandatory, but on occasion even hospitals were shown as non-compliant in reporting outbreaks. The fact that more dynamic data sources could have value compared to traditional slow reporting mechanisms has also been recognized in public health, where social media are used as an early warning system for disease outbreaks [14].

\section{Strengths and limitations}

The main strength of this study is the use of dynamic online content from social media and search engine behaviour in combination with an official reference (SO-ZI/AMR). Using predefined selection criteria, this allowed us to efficiently study the value of social media and online search behaviour via both Coosto and Google Trends. Coosto on occasion is limited by the fact that it may not always be possible to determine whether a potential outbreak is actually a true outbreak. Google Trends has even more difficulty in this determination, for using this data source, it is hard to link potential outbreaks to specific organizations, since it does not provide specific names or locations. A refinement of search mechanism of the freely available default Google Trends software might allow an increase of its sensitivity and specificity.

The extent of information patients and caregivers get when confronted with an outbreak may influence their search behaviour. If the information is complete and offered right away, as it is customary in many Dutch hospitals, it may become more difficult to detect the outbreak using social media and search engine behaviour.

\section{Conclusions}

Despite several limitations including limited validity, using information from social media and online search behaviour results to detect MRSA outbreaks could be an additional source of information for supervising bodies, particularly when combined in an automated system with real-time updates.

\section{Funding}

This research was supported by the EPI-Net COMBACTE-MAGNET project. We thank the Innovative Medicines Initiative Joint Undertaking for supporting the EPI-Net COMBACTE-MAGNET project (grant agreement number 115737), resources of which include financial contribution from the European Union Seventh Framework Programme (FP7/2007-2013) and European Federation of Pharmaceutical Industries and Associations companies in-kind contribution. The funder had no role in the study design, data collection, analysis, interpretation of data or writing the manuscript.

Availability of data and materials

The dataset used during the current study are available from the corresponding author on reasonable request. 


\section{Authors' contributions}

$\operatorname{TvdB}, M v G$ and AV designed the study, with input from LE and JL and RS. TvdB, PvS, KS and MvG collected and analyzed data. TvdB produced a draft of the manuscript, and LE, JL, RS, JRB, ET, KS, MvG and AV reviewed it at various stages to its final version. All authors read and approved the final manuscript.

\section{Ethics approval and consent to participate}

Since the anonymous data used in this study were derived from the public social media domain without patient involvement, no medical ethical review was needed in the Netherlands. SO-ZI/AMR allowed us to use anonymous (not linked to specific hospital organizations) information from their database.

\section{Competing interests}

AV is Editor-in-Chief of ARIC. All other authors declare that they have no competing interests.

\section{Publisher's Note}

Springer Nature remains neutral with regard to jurisdictional claims in published maps and institutional affiliations.

\section{Author details}

'Radboud REshape Innovation Center, Radboudumc University Medical Center, Geert Grooteplein Zuid 10, 6525, GA, Nijmegen, Netherlands. ${ }^{2}$ VetEffecT, Bilthoven, The Netherlands. ${ }^{3}$ Unidad Clínica de Enfermedades Infecciosas y Microbiología Instituto de Biomedicina de Sevilla (IBiS) /Hospital Universitario Virgen Macarena / CSIC / Departamento de Medicina, Universidad de Sevilla, Sevilla, Spain. ${ }^{4}$ Division of Infectious Diseases, Tübingen University Hospital, DZIF Center, Tübingen, Germany. ${ }^{5}$ Infectious Diseases, University of Verona, Verona, Italy. ${ }^{6}$ Department of Medical Microbiology, Radboudumc, Nijmegen, The Netherlands. ${ }^{7}$ Department for Health Evidence, Radboud Institute for Health Sciences, Radboudumc, Nijmegen, The Netherlands. ${ }^{8}$ Department of Clinical Microbiology and Infectious Diseases, Canisius-Wilhelmina Hospital, Nijmegen, The Netherlands.

Received: 15 January 2018 Accepted: 15 May 2018

Published online: 30 May 2018

\section{References}

1. WIP-guideline MRSA. https://www.rivm.nl/dsresource?objectid=3f054354-ff4a43ef-91f9-7c6f0417be95. Accessed 30 Aug 2017.

2. WIP-guideline BRMO (Bijzonder Resistente Micro-Organismen). https:/www. rivm.nl/dsresource?objectid=b6b99580-44e2-4b9c-8183-52871e61764f\&type= org\&disposition=inline. Accessed 30 Aug 2017.

3. Van der Bij AK, van Dijk K, Muilwijk J, Thijsen SFT, Notermans DW, de Greeff S. Van de Sande-Bruinsma N. Clinical breakpoint changes and their impact on surveillance of antimicrobial resistance in Escherichia coli causing bacteraemia. Clin Microbiol Infect. 2012;18:E466-74.

4. RNWWS.nl Criteria uitbraak. https://www.nvmm.nl/zorg/uitbraakmeldingen/. Accessed 30 Aug 2017

5. Ginsberg J, Mohebbi MH, Patel RS, Brammer L, Smolinski MS, Brilliant L. Detecting influenza epidemics using search engine query data. Nature. 2009;457:1012-4.

6. Carneiro HA, Mylonakis E. Google trends: a web-based tool for real-time surveillance of disease outbreaks. Clin Infect Dis. 2009;49:1557-64.

7. van de Belt TH, Engelen $L$, Verhoef LM, van der Weide MJ, Schoonhoven L, Kool RB. Using patient experiences on Dutch social media to supervise health care services: exploratory study. J Med Internet Res. 2015;17(1):e7.

8. van der Bij AK, Kardamanidis K, Frakking FN, Bonten MJ. Signaleringsoverleg Ziekenhuisinfecties en Antimicrobiële Resistentie. Nosocomial outbreaks and resistant microorganisms. Ned Tijdschr Geneeskd. 2015;159:A8585.

9. Coosto, Social Media Monitoring, Webcare \& Publishing. Coosto B.V. Eindhoven, The Netherlands. Version 2017 Coosto http://www.coosto.nl. Accessed 30 Aug 2017.

10. Google Trends. Google. http://google.com/trends. Accessed 30 Aug 2017.

11. Nationale Search Engine Monitor (2014) (iProspect en RMI) https://www. iprospect.com/nl/nl/onze-blog/nationale-search-engine-monitor-2014/. Accessed 30 Aug 2017.
12. Liu K, Huang S, Miao ZP, Chen B, Jiang T, Cai G, Jiang Z, Chen Y, Wang Z, Gu H, Chai C. Jiang J identifying potential norovirus epidemics in China via internet surveillance. J Med Internet Res. 2017;19(8):e282.

13. Denecke K, Dolog P, Smrz P. Making use of social media data in public health. In: Proceedings of the 21st international conference on world wide web. Mountain View: ACM. p. 243-6.

14. Denecke K. Health web science, health information science, chapter 11 , social media for health monitoring p 101-108. Switzerland: Springer International Publishing; 2015. https://doi.org/10.1007/978-3-319-20582-3_11.

\section{Ready to submit your research? Choose BMC and benefit from:}

- fast, convenient online submission

- thorough peer review by experienced researchers in your field

- rapid publication on acceptance

- support for research data, including large and complex data types

- gold Open Access which fosters wider collaboration and increased citations

- maximum visibility for your research: over $100 \mathrm{M}$ website views per year

At BMC, research is always in progress.

Learn more biomedcentral.com/submissions 